\title{
Cylinders vs. Spheres: Biofluid Shear Thinning in Driven Nanoparticle Transport
}

\author{
Jeremy A. Cribb ${ }^{1}$, Timothy D. Meehan², Sheel M. Shah ${ }^{3}$, Kwan Skinner ${ }^{3}$, and Richard \\ Superfine ${ }^{3}$ \\ ${ }^{1}$ Department of Biomedical Engineering, University of North Carolina at Chapel Hill, Chapel Hill, \\ NC, USA \\ ${ }^{2}$ Australian Institute for Bioengineering and Nanotechnology (AIBN), University of Queensland, \\ St. Lucia, QLD 4072, Australia \\ ${ }^{3}$ Department of Physics and Astronomy, University of North Carolina at Chapel Hill, 141 Phillips \\ Hall, CB\# 3255, Chapel Hill, NC 27599, USA
}

\begin{abstract}
Increasingly, the research community applies magnetophoresis to micro and nanoscale particles for drug delivery applications and the nanoscale rheological characterization of complex biological materials. Of particular interest is the design and transport of these magnetic particles through entangled polymeric fluids commonly found in biological systems. We report the magnetophoretic transport of spherical and rod-shaped particles through viscoelastic, entangled solutions using lambda-phage DNA ( $\lambda$-DNA) as a model system. In order to understand and predict the observed phenomena, we fully characterize three fundamental components: the magnetic field and field gradient, the shape and magnetic properties of the probe particles, and the macroscopic rheology of the solution. Particle velocities obtained in Newtonian solutions correspond to macroscale rheology, with forces calculated via Stokes Law. In $\lambda$-DNA solutions, nanorod velocities are 100 times larger than predicted by measured zero-shear viscosity. These results are consistent with particles experiencing transport through a shear thinning fluid, indicating magnetically driven transport in shear thinning may be especially effective and favor narrow diameter, high aspect ratio particles. A complete framework for designing single-particle magnetic-based delivery systems results when we combine a quantified magnetic system with qualified particles embedded in a characterized viscoelastic medium.
\end{abstract}

\section{Keywords}

Shape effects; Drug delivery; Nanorods; Nanowires; Microrheology; DNA; Magnetic bead rheology

\section{INTRODUCTION}

Micro and nanoscale magnetic particles attract much attention in research as probes that reveal the microrheological properties of biological polymer systems and as potential drug

(C) 2010 Biomedical Engineering Society

Address correspondence to Richard Superfine, Department of Physics and Astronomy, University of North Carolina at Chapel Hill, 141 Phillips Hall, CB\# 3255, Chapel Hill, NC 27599, USA. rsuper@ physics.unc.edu.

Jeremy Cribb and Timothy D. Meehan contributed equally to the work.

ELECTRONIC SUPPLEMENTARY MATERIAL The online version of this article (doi:10.1007/s10439-010-0084-5) contains supplementary material, which is available to authorized users. 
carriers in clinical settings. Because of this, the use of magnetophoresis is ever increasing, becoming more common in diagnostic and analytical devices, ${ }^{17}$ general research techniques, ${ }^{1,10,24}$ and clinical applications. ${ }^{33}$ Owing to rapid development, this latter area of medical science employs magnetophoretic systems for drug delivery, $3,28,36$ gene transfection, ${ }^{7}$ and hyperthermic therapies, ${ }^{11,38}$ where the delivery of a particle to a specific target is critical. Materials encountered in these biomedical applications such as tissue, extracellular matrix (ECM), cytoplasm, and synovial fluid contain complex microstructures, which challenge particle transport. Confined paths in dense meshes necessitate the use of nanoscale particles, where the flexibility of the mesh in viscoelastic solids or liquids results in complex rheological behavior and requires the consideration of high shear rate effects. For example, where some biomaterials such as the ECM exist as viscoelastic solids (gel), synovial fluid is an entangled polymer system that exhibits yield phenomena and shear thinning. ${ }^{20}$ Furthermore, the question of particle shape arises as one balances considerations of drug loading, force generation, and transport (drag) effects. Solving these challenges will open new opportunities for the transport of particles and for applications of magnetophoresis in medical science.

The use of magnetophoresis to manipulate particles predictably in these challenging biological environments demands a quantitative understanding of the forces required to produce transport. Despite the increased use of magnetophoresis over a broad range of fields, limited studies have investigated quantitative microparticle magnetophoresis within the context of a well-characterized system. ${ }^{1}$ To date, there are no articles in microstructured biological media relevant to practical biomedical applications that establish full characterizations of applied forces, particle and media properties, alongside single particle measurements. Kuhn et al. showed effective transport for a solution of magnetic particles with observations at the macroscale that measured bulk fluid transport. ${ }^{22}$ In a later article, Kuhn et al. focused on the effects of the particle chemistry and showed increased rates of transport for nanoparticles functionalized with a proteolytic colleganase. ${ }^{21}$ The aim of this study is to develop a predictive understanding of particle magnetotransport that may be used for the design of drug delivery carriers in medical applications. To that end, we require wellcharacterized biofluid media, particles, and magnetic fields as well as single particle transport measurements.

The concentrated solutions of lambda-phage DNA ( $\lambda$-DNA) used here function as useful models of entangled networks typically found in biological media and exhibit complex rheological phenomena. DNA has several advantages over other biopolymer systems that might otherwise be suitable models. It contains a highly monodispersed distribution of polymer lengths which is atypical in synthetic polymer systems, allowing for predictable and reproducible rheological properties from one DNA sample to the next. DNA has a persistence length that is intermediate between synovial fluid and the filaments of ECM (collagen) and cytoplasm (actin, microtubules). In addition, it serves as a model for infected mucus which may contain a high percentage of entangled DNA. ${ }^{32}$ For the purposes of this article, the measured macroscale rheological properties of the $\lambda$-DNA solutions are sufficient to explain our transport data. Using these rheological properties, we can predict the forces required to move a sphere or rod through a shear thinning material like $\lambda$-DNA. For probe particles, we used commercial micron diameter spheres and fabricated nanorods with nominal diameters of $200 \mathrm{~nm}$. We characterized the latter with regard to shape and size using scanning electron microscopy, and measured their magnetic properties using SQUID magnetometry. Finally, we implemented a simple magnetics system with a characterized field and field gradient whose magnitudes are suitable for deployment in medical applications. 
For quantitative magnetophoresis and the understanding of particle shape effects, we first show that calculated and measured transport velocities agree in the transport of the microbeads and the nanorods moving in a Newtonian viscosity standard. Following calibration of the magnetophoretic system, we investigated particle transport in $\lambda$-DNA solutions at two concentrations within the entanglement regime. Particles traveled at velocities over 100 times faster than expected based on Stokes Law and the low shear rate viscosity of the $\lambda$-DNA solutions. We inferred the viscosities from the calculated forces and measured velocities, and determined that the viscosities seen by the particles decreased with increasing particle velocity. This phenomenon is consistent with our measurements of shear thinning in $\lambda$-DNA solutions, and results when an applied shear stress aligns the polymer strands in a semi-dilute or entangled network ${ }^{16}$ or disrupts the size and arrangement of aggregate polymer strands in solution. ${ }^{31}$ While shear thinning has been measured for $\lambda$ DNA using bulk rheological techniques, ${ }^{13}$ this is the first article on shear thinning of a polymer network induced by submicron-sized driven particles. These results have profound implications regarding transport in biomaterials where a short delivery time is a common requirement. Such applications often have practical constraints where the magnitude of driving forces is limited. Demonstrating that particles can induce shear thinning at the submicron scale reduces the force requirements for a magnetophoresis system or might provide faster transport over greater distances than one might otherwise anticipate.

\section{METHODOLOGY}

This article describes the motion of magnetic particles under an applied magnetic field. The force balance $F_{\mathrm{m}}=F_{\mathrm{d}}$ describes particle dynamics in low Reynolds number situations where $F_{\mathrm{m}}$ is the applied magnetic force, and $F_{\mathrm{d}}$ is the drag force due to the fluid. The former depends on particle magnetic properties, and the magnitude and gradient of the magnetic field. The latter depends on the medium properties and the particle geometry. We first describe particle magnetic properties, followed by a characterization of medium properties and particle geometry. The characterization of these quantities follows thereafter.

\section{Nanoparticles}

The particles used in this study were non-magnetic $1 \mu \mathrm{m}$ polystyrene control beads (Polysciences, Warrington, PA), superparamagnetic $1 \mu \mathrm{m}$ carboxy-functionalized MyOne ${ }^{\circledR}$ microspheres (Invitrogen, Carlsbad, CA) and nickel rods fabricated in house. Highly monodisperse, the MyOne bead radius was $525 \mathrm{~nm}$, as reported by the manufacturer and confirmed by our in-house SEM measurements. We used electrochemical deposition to fabricate nickel rods inside $200 \mathrm{~nm}$ pores in a commercially available anodized alumina oxide (AAO) template (Whatman, Maidstone, UK). The nickel rods varied in length from 5 to $20 \mu \mathrm{m}$. This range in rod length provided us with a range of aspect ratios, none of which was below 15 . These large aspect ratios allowed us to approximate a nanorod as a prolate ellipsoid to estimate the magnetomotive force induced by the source magnet and the shear rate along the surface of the rod. We adopted the electrochemical plating bath and deposition parameters from a method devised by Neilsch et al. ${ }^{27}$ After dissolving the membrane, ultrasonication suspended the freed rods into a $0.01 \%$ SDS solution that we later exposed to a magnetic field in excess of $0.4 \mathrm{~T}$.

\section{Magnetic Characterization of Nanoparticles}

A Superconducting Quantum Interference Device (SQUID) magnetometer (Quantum Design, Inc., MPMS-5S) measured the magnetic characteristics of the beads and rods at room temperature. Because their long axis was always parallel to the applied magnetic field during experiments, we characterized the nickel rods as a vertically aligned array while 
being still embedded inside their host template. We measured the physical dimensions of the particles using electron and optical microscopy.

\section{Newtonian Fluid and Viscoelastic $\lambda$-DNA Solution}

Needing a viscous, Newtonian standard to calibrate the magnetophoretic system, we used Light Karo Syrup (ACH Food Companies, Inc.), a commercial product derived from corn. For our viscoelastic polymer system, we prepared solutions of $\lambda$-DNA (Invitrogen, Carlsbad, $\mathrm{CA}$ ), first being careful to anneal one of the single-stranded, hanging ends with a complementary 12 base pair oligonucleotide (UNC oligonucleotide synthesis facility, Chapel Hill, NC), which prevented the formation of cyclic DNA, and minimized potential solution heterogeneity. ${ }^{5}$

A solution of $\lambda$-DNA reaches its overlap concentration at $0.07 \mathrm{mg} / \mathrm{mL}$, and its entanglement concentration is roughly 10 times that at $0.7 \mathrm{mg} / \mathrm{mL} .{ }^{40} \mathrm{We}$ prepared all $\lambda$-DNA solutions from a stock solution of $2.4 \mathrm{mg} / \mathrm{mL}$. Test conditions for nanoparticle experiments included two $\lambda$-DNA solutions within the entanglement regime at 0.7 and $2.0 \mathrm{mg} / \mathrm{mL}$. Owing to the large quantity of solution needed for cone and plate (CAP) rheometry, we restricted the macroscale viscometry measurements to a solution at 1.4 and $0.7 \mathrm{mg} / \mathrm{mL}$. The agreement between our data and literature values and the consistency of the concentration-dependent rheology of $\lambda$-DNA solutions supported this limited application of CAP. We checked for non-specific adsorption of $\lambda$-DNA to the particles, which might confound driven transport, by imaging suspensions of particles dispersed in $\lambda$-DNA, fluorescently labeled with YOYO-1 (data not shown). We observed no significant increase in fluorescence near these particles, indicating little $\lambda$-DNA adsorption (Table 1).

\section{Viscometry of Transport Media}

A controlled-stress cone and plate rheometer (TA Instruments, model AR-G2) measured the viscometry of the Newtonian and $\lambda$-DNA solutions at $23^{\circ} \mathrm{C}$ with a $40 \mathrm{~mm}, 1^{\circ}$ cone across feedback-controlled input shear rates ranging from 1 to $1000 \mathrm{~s}^{-1}$. We fit the viscometry data for the $\lambda$-DNA solutions to a Carreau-Yasuda model,

$$
\frac{\eta-\eta_{\infty}}{\eta_{0}-\eta_{\infty}}=\left[1+(\lambda \dot{\gamma})^{a}\right]^{(n-1) / a}
$$

where $\eta_{0}$ and $\eta_{\infty}$ are the medium viscosities at zero and infinite shear, respectively, $\lambda$ is the thinning time constant, $a$ modulates the width of the thinning regime, and $n$ is the flowbehavior index that leads to the power-law slope equal to $(n-1) \cdot{ }^{13}$ Following the method used by Heo and Larson, we tried first to fit our data to a Cross model, which is oneparameter simpler than the Carreau-Yasuda model for shear thinning. We found that whereas the Carreau-Yasuda model provided sufficient parameters to fit the observed behavior, the Cross model did not. Also, because we compared our data with Heo and Larson, we chose the Carreau-Yasuda model for consistency.

\section{Microparticle Magnetophoresis Apparatus}

The microparticle magnetophoresis apparatus consisted of a 1-in.-long cylindrical rare earth $(\mathrm{NdFeB})$ permanent magnet (K\&J Magnetics, Inc.) mounted on a translation stage and an inverted optical microscope. The sample, enclosed in a transparent microfluidic chamber, contained nanoparticles dispersed in a small volume of test fluid (Fig. 1, inset). Adjustments made to the axial position of the magnet with respect to the sample exposed the particles to variable forces during magnetophoresis experiments. We used a digital Gauss/Teslameter Model 5080 (W. Bell, Orlando, FL) to measure the magnitude of the magnetic field applied to the particles as a function of distance from the axial face of the magnet. We determined 
particle positions by taking time-lapse images of the driven particles using a microscope. The image-acquisition system consisted of a Pulnix camera, model PTM-6710CL (JAI, Inc., San Jose, CA), an EDT-PCI DV (Engineering Design Team, Beaverton, OR) frame grabber card, and a PC workstation. The CISMM Video Spot Tracker software (http:// cismm.cs.unc.edu/downloads/) handled particle tracking while MATLAB routines computed velocities. We mounted the entire experimental apparatus onto a floating optics table to minimize mechanical vibrations.

A microliter-sized volume chamber contained the sample of particles dispersed in the media. We constructed a sealed sample well from a $50-\mu \mathrm{m}$-thick sheet of polydimethylsiloxane (PDMS) containing a 1.5-mm-round hole and two glass coverslips. The diameter of the sample well was less than the width of the magnet to minimize lateral variations of the magnetic field within the sample chamber. Before applying any magnetic field and collecting data, the sample rested for at least $10 \mathrm{~min}$ to ensure the sample reached hydrodynamic equilibrium. Each particle suspension contained non-magnetic particles as well as magnetic particles, enabling a quantitative measure of remaining drift. We subtracted the velocities of the control particles to correct magnetically-driven particle velocities.

\section{Modeling and Measuring Field and Field Gradient}

Deriving from first principles, the magnitude of the magnetic field $B_{\mathrm{m}}$ of a cylindrical source as a function of the axial distance from the face is

$$
B_{\mathrm{m}}(z)=\frac{\mu_{0} M_{0}}{2}\left[\frac{L_{\mathrm{m}}-z}{\sqrt{R_{\mathrm{m}}^{2}+\left(z-L_{\mathrm{m}}\right)^{2}}}+\frac{z}{\sqrt{R_{\mathrm{m}}^{2}+z^{2}}}\right]
$$

where $M_{\mathrm{S}}$ is the magnetic saturation of the magnet, $z$ is the axial distance from the face of the magnet, $L_{\mathrm{m}}$ is the length of the magnet, and $R_{\mathrm{m}}$ is the magnet radius. We measured $B_{\mathrm{m}}$ of the permanent magnet as a function of $z$ from the face of the magnet (Fig. 1), collecting values at lengths that ranged from the magnet face to $18 \mathrm{~mm}$ away. During magnetophoresis experiments, the nanoparticles were always within a range of 3-10 $\mathrm{mm}$ from the magnet face (Fig. 1).

Using least-squares regression, we fit the magnetic field as a function of distance in the $z$ direction, $B_{\mathrm{m}}(z)$, over the experimentally relevant $z$ range (Eq. 2 ). We calculated the axial distance-dependent field gradient of the magnet in the $z$ direction, $\vec{\nabla}_{z} B$, by taking the derivative of Eq. (2) with respect to $z$ (Eq. 3) and substituting the variable values obtained through the fit procedure.

$$
\begin{aligned}
\nabla_{\mathrm{z}} B= & \frac{d B_{\mathrm{m}}(z)}{d x} \\
= & \frac{\mu_{0} M_{0}}{2}\left[\frac{-z^{2}}{\left(R_{\mathrm{m}}^{2}+z^{2}\right)^{3 / 2}}+\frac{1}{\sqrt{R_{\mathrm{m}}^{2}+z^{2}}}\right. \\
& \left.+\frac{\left(L_{\mathrm{m}}-z\right)^{2}}{\left(R_{\mathrm{m}}^{2}+\left(z-L_{\mathrm{m}}\right)^{2}\right)^{3 / 2}}-\frac{1}{\sqrt{R_{\mathrm{m}}^{2}+\left(z-L_{\mathrm{m}}\right)^{2}}}\right]
\end{aligned}
$$

\section{Quantitative Microparticle Magnetophoresis}

We describe the magnetic driving forces, $\vec{F}_{\mathrm{m}}$, applied to the particles in this study as

$$
\vec{F}_{\mathrm{m}}=\left(\vec{m}_{\mathrm{eff}} \cdot \vec{\nabla}\right) \vec{B}
$$


where the effective magnetization of the particles $\vec{m}_{\mathrm{eff}}$ is a function of the magnetic induction $\vec{B}$ and the magnetic properties of the particles. In addition to $\vec{m}_{\mathrm{eff}}, \vec{F}_{\mathrm{m}}$ is a function of $\vec{\nabla} B$. Under the experimental conditions of this study, $\vec{F}_{\mathrm{m}}$ is, therefore, proportional to $\vec{B}$ and $\vec{\nabla} B$. The relation that defines the magnetic force on a sphere ${ }^{9,26}$ is

$$
F_{\mathrm{s}}=\frac{\left(\mu_{\mathrm{r}}-1\right)}{2 \mu_{0}} \cdot \frac{3}{8\left(\mu_{\mathrm{r}}+2\right)} \cdot V_{\mathrm{s}} \cdot \nabla\left(B^{2}\right)
$$

where $V_{\mathrm{s}}$ and $\mu_{\mathrm{r}}$ are the volume and the relative permeability of the sphere, respectively. We use the standard definition of relative permeability, $\mu_{\mathrm{r}}=\mu / \mu_{0}$, which is the ratio of material permeability to the permeability of free space. We can show that the force exerted on a cylinder, $F_{\mathrm{c}}$, approximated by a prolate ellipsoid with an aspect ratio greater than 20 , is

$$
F_{\mathrm{c}}=\frac{\left(\mu_{\mathrm{r}}-1\right)}{2 \mu_{0}} \cdot V_{\mathrm{c}} \cdot \nabla\left(B^{2}\right)
$$

where $V_{\mathrm{c}}$ is the cylindrical volume, and $\mu_{\mathrm{r}}$ is the relative permeability of the magnetic material. ${ }^{29}$

We performed the driven particle transport described here under low Reynolds number conditions $(R e \ll 1)$, enabling the correlation of the magnetic driving forces, particle geometries, and velocities in a Newtonian fluid (corn syrup) through Stokes' Law. We compared these empirical magnetophoretic forces with predicted values that were based on the independently measured magnetic characteristics of the system. Owing to the low Reynolds number conditions, we set $\vec{F}_{\mathrm{m}}$ equal to the magnitude of the frictional drag force on the particle, $\vec{F}_{\mathrm{d}}$.

In general, the frictional Stokes drag force on a particle under such conditions is $\vec{F}_{\mathrm{d}}=-\beta \eta \nu$, where $v$ is the particle velocity, $\eta$ is the medium viscosity, and $\beta$ is the geometry coefficient specific to the particle geometry. This relation enables the determination of the apparent viscosities, $\eta_{\text {app }}$, of the $\lambda$-DNA solutions as experienced by the magnetically driven particles. For a spherical geometry with radius $r_{\mathrm{s}}$, the geometry coefficient, $\beta_{\mathrm{s}}$, is simply

$$
\beta_{\mathrm{s}}=6 \pi r_{\mathrm{s}} \quad \text { (7) }
$$

Approximated by Tirado and de la Torre, the geometry coefficient, $\beta_{\mathrm{c}}$, for an axially translating cylindrical rod with an aspect ratio greater than 2 is

$$
\beta_{\mathrm{c}}=\frac{2 \pi L}{\ln \left(\frac{L}{2 r_{\mathrm{c}}}\right)+\gamma_{\|}}
$$

where $r_{\mathrm{c}}$ is the rod radius, $L$ is the length of the rod, and $\eta_{\|}$is equal to -0.19 , as an end correction factor. ${ }^{37}$ Rod motion was always in the axial direction, because the rods align naturally along their axis length to the field lines that emanate from the drive magnet. The nickel rods had radii between 100 and $175 \mathrm{~nm}$ and a range of different lengths due to breakage during processing which required an individual calculation of $\beta_{\mathrm{c}}$ for each rod. Error analysis indicates this range of rod radii would add $10 \%$ variability to the viscosity results. 
One typically applies Stokes Law in particle transport experiments to deduce the applied force on a particle, with independent coefficients for particle geometry and fluid viscosity. In order to use Stokes Law to measure fluid viscosity, we need to know the force applied to the particle. We obtain this in two ways and show their agreement. First, we compute $\vec{F}_{\mathrm{m}}(z)$ for the particles based on Eqs. (5) and (6). Second, we obtain the applied particle force using $\vec{F}_{\mathrm{d}}=-\beta \eta \nu$, with measured values of $\eta$ of the Newtonian solution, of the geometries of the particles, and of their measured velocities. We then use the force on the particle as a measured quantity and apply a generalized form of Stokes Law to show that our transport measurements are consistent with the shear rate-dependent viscosity of the $\lambda$-DNA solutions.

\section{Determination of Microscale Shear Rate}

For the conversion of bead velocity to shear rate in a viscous fluid, we use the azimuthal velocity, $v_{\theta}$ of the fluid with respect to the bead

$$
\nu_{\theta}=\nu_{\mathrm{d}} \sin \theta\left(1-\frac{3 r_{\mathrm{s}}}{4 r}-\frac{r_{\mathrm{s}}^{3}}{4 r^{3}}\right)
$$

where $r_{\mathrm{s}}$ is the sphere's radius, $r$ and $\theta$ are the radial and angular coordinates for a fluid parcel that moves with respect to the sphere. ${ }^{2}$ We find the maximum shear rate component of the strain rate tensor for a spherical geometry by differentiating $v_{\theta}$ with respect to $r$ and setting $r=r_{\mathrm{S}}$, and $\theta=\pi / 2$. Taking the norm of the tensor yields ${ }^{35}$

$$
\left|\dot{\gamma}_{\mathrm{s}}\right|=\frac{3 \nu_{\mathrm{s}}}{\sqrt{2} r_{\mathrm{s}}}
$$

and similarly, in order to estimate the shear rate along the surface of the nickel rods, we derived the shear rate magnitude at the top of a prolate ellipsoid (derivation available as supplemental information) to be

$$
\left|\dot{\gamma}_{\mathrm{c}}\right|=\frac{2 \sqrt{2} \nu_{\mathrm{c}}}{r_{\mathrm{c}}(-1+\ln (4)+2 \ln (p))}
$$

where $r_{\mathrm{c}}$ is the length of the minor axis, and $p=L / 2 r_{\mathrm{c}}$ is the aspect ratio of the cylinder. Data from the CAP studies of the $\lambda$-DNA solution were used to understand the enhanced transport seen by our nanoparticles. In order to place our measurements within the shear thinning

regime, the Stokes equation is written as $\eta=-\vec{F}_{\mathrm{d}} / \beta \nu$, and the velocity is written in terms of the shear rate according to Eqs. (10) and (11). Finally, we equate the drag and magnetic forces to obtain a form of Stokes Law that explicitly contains the shear rate:

$$
\begin{gathered}
\eta_{\text {sphere }}(\dot{\gamma})=\frac{3 F_{\mathrm{m}, \text { sphere }}}{\sqrt{2} r \beta_{\mathrm{s}} \dot{\gamma}} \\
\eta_{\text {cylinder }}(\dot{\gamma})=\frac{2 \sqrt{2} F_{\mathrm{m}, \text { cylinder }}}{L(-1+\ln (4)+2 \ln (p)) \beta_{\mathrm{c}} \dot{\gamma}}
\end{gathered}
$$

This allows us to plot our transport data in direct comparison with the Carreau-Yasuda model fits to viscometry data. 


\section{Shape Effect Attributes}

Particle Velocity vs. Shape-The delivery speed of a carrier particle and the number of deliverable molecules are of paramount significance when one designs a magnetophoretic drug delivery system because both characteristics depend on particle size and shape. The magnetophoretic velocity results from a combination of driving and drag forces (Eqs. 12 and 13) applied to the particle, which are related to the volume of magnetized material and the drag coefficients, respectively.

$$
\frac{\nu_{\mathrm{c}}}{\nu_{\mathrm{s}}}=\left(\frac{\beta_{\mathrm{s}}}{\beta_{\mathrm{c}}}\right) \cdot\left(\frac{F_{\mathrm{c}}}{F_{\mathrm{s}}}\right) \cdot \frac{\eta_{\mathrm{s}}\left(\dot{\gamma}_{\mathrm{s}}\right)}{\eta_{\mathrm{c}}\left(\dot{\gamma}_{\mathrm{c}}\right)}
$$

Attached to the surface of delivery particles and/or loaded internally, the number of deliverable payload molecules is a function of the surface area or volume of the particle.

Drag Force vs. Shape-We now compare the choice between spherical and cylindrical nanoparticles for transport. Under the assumption that both particle shapes studied here have equal volume, the relationship between the radius of a sphere and the radius and axial length of a cylinder is $r_{s}=(3 L / 4)^{1 / 3} r_{\mathrm{c}}^{2 / 3}$, which shows a higher sensitivity to $r_{\mathrm{c}}$ over $L$. Given a constant particle velocity and Newtonian viscosity, we compute the ratio of the geometry coefficients as

$$
\frac{\beta_{\mathrm{c}}}{\beta_{\mathrm{s}}}=\left(\frac{81}{16}\right)^{-1 / 3} p^{2 / 3}\left(\ln (p)+\gamma_{\|}\right)^{-1}
$$

For $p>1$, we find that $\beta_{\mathrm{c}} / \beta_{\mathrm{s}}>1$, resulting in a drag penalty when one switches from a spherical to a cylindrical shape. For our cylinders $p$ ranges from 15 to 105 , corresponding to an increase in drag of up to $65 \%\left(\beta_{\mathrm{c}} / \beta_{\mathrm{s}} \sim 1.65\right)$ for the cylinder over that of the sphere.

Magnetic Force vs. Shape-Given the smaller viscous drag coefficient of a sphere vs. that of an equal volume rod, a researcher might conclude that a spherical particle would represent the best option for delivery in a biomedical context. However, one must also consider the effect shape has on the driving force, which does not depend solely on magnetic content.

When comparing the forces on a sphere (Eq. 5) and cylinder (Eq. 6) made of the same material and equal volume, the force ratio is

$$
\frac{F_{\mathrm{c}}}{F_{\mathrm{s}}}=\frac{8\left(\mu_{\mathrm{r}}+2\right)}{3} \text {. }
$$

This ratio must be greater than 1 when using any magnetic material, implying that for these conditions, $F_{\mathrm{c}}>F_{\mathrm{s}}$ by the factor shown above. For our nickel nanorods, where $\mu_{\mathrm{r}}=18.3$, this results in an applied force that is about 50 times higher for a cylinder than for a sphere with the same volume.

Viscosity vs. Shear Rate-Because the spheres and cylinders experience different shear rates, we must recognize the concomitant change in the shear rate-dependent viscosity and compare the viscosity ratio, $\eta_{\mathrm{c}}\left(\gamma_{\mathrm{c}}\right) \eta_{\mathrm{s}}\left(\gamma_{\mathrm{s}}\right)$, via the Carreau-Yasuda model. Because $\eta_{0} \gg \eta_{\infty}$, we can approximate $\eta_{\infty}$ as zero, and because we focus on just the high shear rates in the thinning regime, we can further simplify the ratio to 


$$
\frac{\eta_{\mathrm{c}}\left(\dot{\gamma}_{\mathrm{c}}\right)}{\eta_{\mathrm{s}}\left(\dot{\gamma}_{\mathrm{s}}\right)} \approx\left(\frac{\dot{\gamma}_{\mathrm{c}}}{\dot{\gamma}_{\mathrm{s}}}\right)^{n-1}
$$

which incorporates an error of $5 \%$ for our experiments.

When we combine the effects of magnetic and drag forces (Table 2), we can compute the composite ratio of particle velocities within $10 \%$ error with

$$
\frac{\nu_{\mathrm{c}}}{\nu_{\mathrm{s}}}=\frac{3 p^{2 / 3}\left(\mu_{\mathrm{r}}+2\right)}{2\left(\ln (p)+\nu_{\|}\right)}\left(\frac{\dot{\gamma}_{\mathrm{c}}}{\dot{\gamma}_{s}}\right)^{n-1}
$$

The velocity ratios for the types of particles used in our experiments show a clear bias for the rod geometry, with approximately 200 times the effective transport rate. The primary contributor to the effectiveness of rod transport is the force ratio, with the drag and viscosity ratios mostly canceling each other out.

Shear Rate vs. Shape-Because the rod experiences a larger force than a sphere of equal volume, it has the potential to experience greater shear thinning due to a higher drift velocity and therefore a higher shear rate. The $\gamma_{\mathrm{c}} / \gamma_{\mathrm{s}}$ ratio shows dependence upon the aspect ratio as well as the flow behavior index, $n$.

$$
\dot{\gamma}_{\mathrm{c}} / \dot{\gamma}_{\mathrm{s}}=\left[(12)^{1 / 3} \frac{p\left(\mu_{\mathrm{r}}+2\right)}{\left(\ln (p)+\gamma_{\|}\right)(-1+\ln (4)+2 \ln (p))}\right]^{1 /(2-n)}
$$

We used the range of aspect ratios found in our experiments to compute ratios that range from 7 to 11 . These $\gamma_{\mathrm{c}} \gamma_{\mathrm{s}}$ ratios indicate a 7-11-fold increase in the shear rate for the cylinder over the sphere. It is important to note that the cylinder's high shear rate advantage vanishes for a Newtonian fluid as it lacks shear rate dependence. In a shear thinning fluid, however, this increase in the shear rate results in a greater velocity for the rod compared to the sphere. Access to these higher shear rates allows a rod shaped particle to propagate almost 10 times more effectively through a lower viscosity medium. We note that a rodshaped particle might have additional advantages over a bead in its ability to penetrate the interstitial spaces and entanglements between polymer molecules in biomaterials, though we do not explore these advantages in this article.

\section{RESULTS}

\section{Solution Viscometry}

The Newtonian solution used in this investigation had a zero-shear viscosity of $3.38 \pm 0.04$ $\mathrm{Pa} \mathrm{s}$ and was constant for any input $\gamma$ below $100 \mathrm{~s}^{-1}$ (Fig. 2). The particles driven in this solution had $\gamma$ values less than $100 \mathrm{~s}^{-1}$. In contrast, CAP rheometry of a $1.4 \mathrm{mg} / \mathrm{mL} \lambda$-DNA solution revealed significant shear thinning at shear rates greater than $0.14 \mathrm{~s}^{-1}$, which corresponds to a $\lambda$ of $7 \mathrm{~s}$. The thinning regime extended to the highest shear rates tested, i.e., $20 \mathrm{~s}^{-1}$. The power-law slope for the thinning regime was -0.96 , leaving $n=0.04$. Both of these values for the Carreau-Yasuda model parameters agree well when compared to data published in Heo and Larson. ${ }^{13}$ Our data from CAP rheometry of $0.7 \mathrm{mg} / \mathrm{mL} \lambda$-DNA solution showed less than $8 \%$ average variability when compared to the data published in Heo and Larson. ${ }^{13}$ In light of the agreement of our CAP study with published values and 
because of the expense of $\lambda$-DNA at the required concentrations and volumes, we fit powerlaw slopes to the tested concentrations in Heo and Larson ${ }^{13}$ and extrapolated to a slope for our microscale tested concentration at $2 \mathrm{mg} / \mathrm{mL}$. In order to understand the effect temperature might have on particle transport through the viscoelastic medium, we increased the temperature of the $0.7 \mathrm{mg} / \mathrm{mL} \lambda$-DNA solution to $37^{\circ} \mathrm{C}$. The zero-shear viscosity for the warmer solution was roughly one-half the value of the cooler solution. At higher shear rates, where shear thinning modes dominate, both rheology measurements converged to a common relationship independent of both temperatures. The effect that solution temperature had on particle transport is explained in more detail in the supplementary information.

\section{Nanoparticle Magnetic Properties}

Under experimental conditions, the maximum magnetic field experienced by the particles in our magnetophoresis system was less than $0.06 \mathrm{~T}$, as determined by measurements with a Hall probe in our permanent magnet system. We obtained excellent fits for the expected position dependence of the magnetic field for the permanent cylindrical magnet (Fig. 1). This value sets the relevant range of magnetic fields for the magnetometry measurements of the microbeads and nanorods.

We measured the magnetic characteristics of the particles to enable the calculation of the magnetic driving forces applied during magnetophoresis experiments. Using the SQUID magnetometry data, we determined that, at low fields, the volumetric susceptibility of MyOne beads was $0.86 \pm 0.02$ (Fig. 3). From these measurements, the MyOne beads magnetically saturated at an applied field of $0.5 \mathrm{~T}$ and had a saturation magnetization $M_{\text {sat }}$ of $53 \pm 1 \mathrm{kA} / \mathrm{m}$.

For the nickel nanorods, we found the volumetric susceptibility was $18.3 \pm 0.3$ based on magnetometry measurements and the pore density of the membrane (Fig. 3). This value incorporates a normalization factor ${ }^{26}$ derived from articles of the magnetic characterization of similar rods by other researchers $6,8,15,25$ and accounts for the influence of the rod packing density on the measured magnetization. In addition to the magnetization induced by the applied field, the rods showed some ferromagnetic character which resulted in a remanent magnetization of $39 \pm 2 \mathrm{kA} / \mathrm{m}$. The rods saturated at an applied field of $0.4 \mathrm{~T}$ and had a $M_{\text {sat }}$ value of $590 \pm 30 \mathrm{kA} / \mathrm{m}$.

\section{Magnetic Forces on Particles}

Since the magnetic particles did not saturate in our transport measurements, we calculated $\vec{F}_{\mathrm{m}}$ using the magnetic characteristics of the particles and the applied magnetic field (Eqs. 5 and 6). Because $\vec{F}_{\mathrm{m}}$ and $\vec{F}_{\mathrm{d}}$ for the rods should be proportional to $L$, we normalized the force values for each rod by dividing out $L$, enabling comparisons between individual rods.

The predicted and experimental values of $\vec{F}_{\mathrm{m}}(z)$ for both the MyOne beads and the rods agreed well within the experimental uncertainties as shown in Fig. 4, which lends confidence to our ability to calculate viscosities of $\lambda$-DNA solutions by solving for the $\eta$ term using Stokes drag.

\section{Driven Particle Transport in Complex Fluids}

In order to understand the nature of driven particle transport in biological fluids, we applied magnetic forces and drove MyOne beads and rods through $\lambda$-DNA solutions at 0.7 and 2.0 $\mathrm{mg} / \mathrm{mL}$. Particles experience two different shape-dependent forces, one imparted by particle magnetization and is proportional to volume, and the other by drag and is proportional to cross-sectional radius and the apparent viscosity, which is also geometry dependent. In order to separate the effects of these two forces on transport, we first plot the particle velocity 
normalized by the shape dependence of the drag coefficient vs. $B(z) \vec{\nabla} B(z)$, the quantity responsible for the driving force (Fig. 5). If the particles experienced only the shapedependent effects on their drag, and experienced the same viscosity, then their curves in the same fluid should lie on top of each other. This is approximately the case for the beads and rods in the Newtonian standard. As expected, particle velocities increase as the magnetic field and field gradient increase. Focusing on the particle transport in the Newtonian standard, we see that the nanorods achieve higher normalized velocities than the microbeads in part due to their higher magnetizations in the same applied fields. We present no data for MyOne beads in $2.0 \mathrm{mg} / \mathrm{mL} \lambda$-DNA because we found no measurable displacement on experimental timescales.

Compared to Newtonian solutions, the relative transport properties of particles are dramatically different when the medium is a viscoelastic $\lambda$-DNA solution. Figure 5 shows that, relative to the velocity of the particles in a Newtonian fluid, the beads experience slower velocities while the nanorods experience faster velocities in $\lambda$-DNA. In $\lambda$-DNA solutions, nanorods move with $>100$ times the bead velocity, regardless of the solution temperature, as opposed to only a fivefold increase when using the Newtonian standard. We interpret the relative differences in the driven velocities as a change in the apparent media viscosity $\eta_{\text {app }}$ experienced by the particles. These differences result from the responses of the media to the shear stresses applied by the driven motion of the particles.

In order to investigate the driven particle induced shear thinning of the $\lambda$-DNA solutions, we plot in Fig. 6 the apparent viscosity, $\eta_{\text {app }}$, as measured by the particles as a function of shear rate. The CAP measurements (shown as red solid lines) reveal the $\lambda$-DNA solution as a shear thinning material at shear rates greater than $\sim 10^{-1} \mathrm{~s}^{-1}$. The nanoparticles (shown as discrete points) roughly reproduce the apparent solution viscosity measured by the CAP irrespective of solution temperature. In conjunction with the maximum shear rate, we plot the measured rod velocity normalized by length (indicated by green axis and arrows), which normalizes for the various rod lengths obtained during fabrication.

In contrast to the behavior of the particle motion in the Newtonian standard, the apparent viscosity of the $\lambda$-DNA solution, as derived from particle motion, indicates a power-law dependence that corresponds to shear thinning of the matrix. In $\lambda$-DNA, power-law fits to the data revealed exponents of -0.8 for rods (both concentrations) and -0.6 for beads $(0.7$ $\mathrm{mg} / \mathrm{mL}$ ). These power-law exponents are consistent with those measured for $\lambda$-DNA by bulk rheological techniques. ${ }^{13}$ As expected in all cases, the values of $\eta_{\text {app }}$ of the $\lambda$-DNA solutions are greater than the viscosity of pure buffer $\left(10^{-3} \mathrm{~Pa} \mathrm{~s}\right)$.

\section{DISCUSSION}

With a quantitative understanding of magnetic particle transport in hand, we now relate these measurements to a set of forces required to deliver particles given the size and shape. In Fig. 6, we show the force per micron requirements for a rod, driven through 0.7 and $2 \mathrm{mg} /$ $\mathrm{mL} \lambda$-DNA solutions (shown in blue, dashed lines), along with the resulting viscosities and their dependence on velocity and shear rate (shown in red). These lines indicate the force necessary to achieve a shear rate (black) or velocity (green) when one expects a given sheardependent viscosity (red). For example, to deliver a rod within the shear thinning region of $\lambda$-DNA at $2.0 \mathrm{mg} / \mathrm{mL}$ with a $10 \mu \mathrm{m}$ rod at $1 \mathrm{~s}^{-1}$, find the shear rate along the $x$-axis and locate the expected viscosity; for this example, the viscosity is $1 \mathrm{~Pa} \mathrm{~s}$, well within the shear thinning regime for this material. At this shear rate, also note the force requirement for each concentration. For $2 \mathrm{mg} / \mathrm{mL} \lambda$-DNA, one applies $\sim 3 \mathrm{pN}$ to achieve the desired shear rate, whereas $0.7 \mathrm{mg} / \mathrm{mL}$ DNA requires only $0.3 \mathrm{pN}$. 
There are a variety of physiological contexts where shear thinning particle delivery may be useful. Here we discuss three: extracellular matrix, mucus barriers, and synovial fluid. In the context of particle delivery through tissue, Lai et al. used canonical CAP methods to measure viscosities for a simulated tissue (bovine-hide collagen) as high as $400 \mathrm{~Pa} \mathrm{~s}^{23}$ Kong and Vazquez measured the viscoelastic properties of gelled collagen used as an extracellular matrix simulant and found shear thinning at $\sim 0.05 \mathrm{~s}^{-1}$ with a zero-shear viscosity at $\sim 1 \mathrm{~Pa} \mathrm{~s} .{ }^{19}$ Particle delivery through mucus is important for pulmonary or cervical delivery of drugs. Besseris and Yeates utilized rotating magnetic micron-sized particles to measure a zero-shear viscosity for canine tracheal mucus that lies between 10 and $7500 \mathrm{~Pa} \mathrm{~s}^{4}$, and measurements by Powell et al. ${ }^{30}$ showed that mucus can thin at shear rates greater than $1 \mathrm{~s}^{-1}$. Our measurements in solutions of DNA are relevant to measurements of mucus rheology because large amounts of DNA are found in pathological (i.e., CF) sputum. ${ }^{32}$ Heo and Larson demonstrated that the application of shear stress results in shear thinning in $\lambda$-DNA at the macro scale where the measured viscosities are diminished by an order of magnitude or more. ${ }^{13}$ Delivery of drugs to or through cartilaginous tissue requires particle transport through synovial fluid and corresponding measurements by Jay et al. ${ }^{18}$ in bovine synovial fluid found thinning at shear rates greater than $0.1 \mathrm{~s}^{-1}$. For the purposes of determining a proper applied force regime for the range of materials introduced above, we first consider the zero-shear viscosity limit. Initially, to achieve a $100 \mathrm{~nm} / \mathrm{s}$ velocity, a 200 -nm-diameter rod would require forces ranging from 0.4 to $300 \mathrm{pN}$ per micron in length, with the lower range of force for synovial fluid and the higher range for mucus. This velocity would correspond to a shear rate of $1.5 \mathrm{~s}^{-1}$, and would be sufficient to engage shear thinning in any of the aforementioned biopolymer systems. A bead with the same volume (and thus equal payload) and made from the same magneticallypermeable material would require 3 to 5 times more force to achieve similar shear rates. In order to achieve this same magnetomotive force, $(B \cdot \nabla) B$ would need to be 75 times higher (assuming no saturation), and therefore requires a larger or closer permanent magnet. Choosing rod-shaped particles as drug carriers becomes obvious when one considers the impact of the lower required magnetic fields and field gradients.

A functioning apparatus may not require very large and unwieldy magnets positioned uncomfortably close to the patient's body. In this article, we used a rare-earth permanent source magnet that was relatively small, (2-mm diameter, 1-in. length) which generated a shear thinning flow for rods at distances as far away as $5 \mathrm{~mm}$ in a fluid with rheological properties comparable to healthy mucus. In terms of source magnet design, the force generated varies minimally with respect to changes in aspect ratio of the magnet. However, increasing the radius of the magnet leads to an increased force and increased penetration depth of the force. In fact, to achieve maximum force at a given depth, a magnet with a radius of about twice the desired depth is required, but the maximum force for a given depth is not necessarily required to achieve significant shear thinning. As the magnetomotive force depends on the product of the field and field gradient, the force generated by a cylindrical magnet scales as $1 / z^{2}$ at distances larger than twice the diameter, where $z$ is distance from the face of the magnet. Our calculations indicate that shear thinning in $2 \mathrm{mg} / \mathrm{mL} \lambda$-DNA can be achieved at depths typically seen in the lung $(\sim 1 \text { in. })^{34}$ with a NdFeB source magnet as small as $20.5 \mathrm{~mm}$ in diameter and $20.5 \mathrm{~mm}$ long. We used the magnetization value of our experimental source magnet to calculate that, a magnet with these specifications, along with the nickel rods of 0.1 and $1.5 \mu \mathrm{m}$ in radius and length, respectively, used in this study, would produce about $0.11 \mathrm{pN}$ of force. As shown in Fig. 6, the force produced would lead to a 100 -fold decrease in viscosity in $2 \mathrm{mg} / \mathrm{mL} \lambda$-DNA, a relevant model for infected mucus. ${ }^{32}$ In the context of a drug delivery system, the velocity of the rod shaped particles would be on the order of $1 \mu \mathrm{m} / \mathrm{s}$ and can, therefore, traverse the approximate length of the mucus layer of the lung epithelia in seconds and the typical length of a mucus plug in a matter of minutes. Without the shear thinning of this material, the same particle would travel at the zero shear 
viscosity, or 1000-fold slower, and therefore take 1000 times longer to reach the desired displacement.

Shear thinning of mucus by these micro-particles opens up a myriad of possibilities in drug delivery, specifically inhalation delivery. Two main pathways exist for the clearance of particles transported through the respiratory epithelium: the mucociliary clearance system and alveolar macrophages. ${ }^{12}$ The internalization of carrier particles by alveolar macrophages has been shown to be heavily dependent on particle size. In fact, using carrier particles smaller than $0.26 \mu \mathrm{m}$ in diameter, which are within the parameters of the experiments described here, avoids macrophageal phagocytosis altogether. ${ }^{14}$ By designing a drug delivery system that takes advantage of the shear thinning of mucus to increase particle transport rate, the problems arising from mucociliary clearance can be obviated, increasing bioavailability of the drug in the lungs. However, in order to get net transport, the particle motion through the mucus layer to the epithelium would need to compete with the mucociliary clearance rate, which in a normal human trachea is $\sim 200 \mu \mathrm{m} / \mathrm{s} .{ }^{39}$ Magneticdriven transport in this case may tilt the balance toward effective transfection.

\section{CONCLUSIONS}

By using solutions of $\lambda$-DNA within the entanglement regime, we demonstrated that the transport of magnetically driven nanoparticles can induce shear thinning of a polymer network. The presence of shear thinning indicates that driven particles experience viscosities in biological materials that are significantly less than the bulk material viscosities as measured under zero-shear conditions. Consequently, predictions based on zero-shear viscosity assumptions alone overestimate the force required to obtain sufficient particle transport. This finding has practical implications for a host of biomedical applications ranging from drug delivery to hyperthermic therapies, where the rapid particle transport with minimal driving force is essential.

We fully characterized the magnetophoretic system used in this study, calibrated it using a Newtonian fluid standard, and confirmed our calibration with SQUID measurements. This enabled us to predict and quantify magnetic driving forces and viscous drag forces on individual particles. We found that the magnetic fields and field gradients required to transport a rod-shaped geometry are easily achieved. Our source was a widely available rare earth $(\mathrm{NdFeB})$ permanent magnet, and the distance between particles and magnet was in the millimeter to centimeter range, a relevant scale of driving forces considered practical for biomedical applications. The combination of a magnetically driven system, fully characterized probe particles, and a non-Newtonian medium comprise a generic framework for performing drug-delivery studies.

\section{Supplementary Material}

Refer to Web version on PubMed Central for supplementary material.

\section{Acknowledgments}

The authors would like to thank staff of Frank Tsui's lab for the SQUID measurements of beads and nanorods, Benjamin Evans for assistance in deriving the force on a prolate ellipsoid, and Leandra Vicci for assistance with COMSOL simulations. We gratefully acknowledge financial support from the National Cancer Institute (the Carolina Center of Cancer Nanotechnology and Excellence) (NIH-5-654-CA119373-02), the NIBIB for CISMM (P41-EB002025-24A1), and 3DFM (R01-EB000761), and the NHLBI for the Virtual Lung project (R01HL077546-01A2). 


\section{References}

1. Amblard F, Yurke B, Pargellis A, Leibler S. A magnetic manipulator for studying local rheology and micromechanical properties of biological systems. Rev Sci Instrum. 1996; 67(3):818-827.

2. Berg, HC. Random Walks in Biology. Princeton, NJ: Princeton University Press; 1993.

3. Berry CC, Curtis ASG. Functionalisation of magnetic nanoparticles for applications in biomedicine. J Phys D Appl Phys. 2003; 36(13):R198-R206.

4. Besseris GJ, Yeates DB. Rotating magnetic particle microrheometry in biopolymer fluid dynamics: mucus microrheology. J Chem Phys. 2007; 127(10):105106. [PubMed: 17867785]

5. Braun E, Eichen Y, Sivan U, Ben-Yoseph G. DNA-templated assembly and electrode attachment of a conducting silver wire. Nature. 1998; 391(6669):775-778. [PubMed: 9486645]

6. Ciureanu M, Beron F, Clime L, Ciureanu P, Yelon A, Ovari TA, Cochrane RW, Normandin F, Veres T. Magnetic properties of electrodeposited $\mathrm{CoFeB}$ thin films and nanowire arrays. Electrochim Acta. 2005; 50(22):4487-4497.

7. Dobson J. Gene therapy progress and prospects: magnetic nanoparticle-based gene delivery. Gene Ther. 2006; 13(4):283-287. [PubMed: 16462855]

8. Encinas-Oropesa A, Demand M, Piraux L, Ebels U, Huynen I. Effect of dipolar interactions on the ferromagnetic resonance properties in arrays of magnetic nanowires. J Appl Phys. 2001; 89(11): 6704-6706.

9. Fisher JK, Cribb J, Desai KV, Vicci L, Wilde B, Keller K, Taylor RM, Haase J, Bloom K, O’Brien ET, et al. Thin-foil magnetic force system for high-numerical-aperture microscopy. Rev Sci Instrum. 2006; 77(2):023702.

10. Gijs MAM. Magnetic bead handling on-chip: new opportunities for analytical applications. Microfluid Nanofluid. 2004; 1(1):22-40.

11. Goya GF, Grazu V, Ibarra MR. Magnetic nanoparticles for cancer therapy. Curr Nanosci. 2008; $4(1): 1-16$.

12. Groneberg DA, Witt C, Wagner U, Chung KF, Fischer A. Fundamentals of pulmonary drug delivery. Respir Med. 2003; 97(4):382-387. [PubMed: 12693798]

13. Heo Y, Larson RG. The scaling of zero-shear viscosities of semidilute polymer solutions with concentration. J Rheol. 2005; 49(5):1117-1128.

14. Holma B. Lung clearance of mono- and di-disperse aerosols determined by profile scanning and whole-body counting. A study on normal and SO2 exposed rabbits. Acta Med Scand. 1967; 473:1-102.

15. Hultgren A, Tanase M, Chen CS, Meyer GJ, Reich DH. Cell manipulation using magnetic nanowires. J Appl Phys. 2003; 93(10):7554-7556.

16. Hyun K, Kim SH, Ahn KH, Lee SJ. Large amplitude oscillatory shear as a way to classify the complex fluids. J Non-Newton Fluid Mech. 2002; 107(1-3):51-65.

17. Jain KK. Nanodiagnostics: application of nanotechnology in molecular diagnostics. Expert Rev Mol Diagn. 2003; 3(2):153-161. [PubMed: 12647993]

18. Jay GD, Torres JR, Warman ML, Laderer MC, Breuer KS. The role of lubricin in the mechanical behavior of synovial fluid. Proc Natl Acad Sci USA. 2007; 104(15):6194-6199. [PubMed: 17404241]

19. Kong QJ, Vazquez M. Flow-induced shear stresses increase the number of cell-cell contacts within extracellular matrix. J Biomed Mater Res Part A. 2009; 89A(4):968-979.

20. Krause WE, Bellomo EG, Colby RH. Rheology of sodium hyaluronate under physiological conditions. Biomacromolecules. 2001; 2(1):65-69. [PubMed: 11749156]

21. Kuhn SJ, Finch SK, Hallahan DE, Giorgio TD. Proteolytic surface functionalization enhances in vitro magnetic nanoparticle mobility through extracellular matrix. Nano Lett. 2006; 6(2):306-312. [PubMed: 16464055]

22. Kuhn SJ, Hallahan DE, Giorgio TD. Characterization of superparamagnetic nanoparticle interactions with extracellular matrix in an in vitro system. Ann Biomed Eng. 2006; 34(1):51-58. [PubMed: 16477503] 
23. Lai GL, Li Y, Li GY. Effect of concentration and temperature on the rheological behavior of collagen solution. Int J Biol Macromol. 2008; 42(3):285-291. [PubMed: 18275999]

24. Lehmann U, Hadjidj S, Parashar VK, Vandevyver C, Rida A, Gijs MAM. Two-dimensional magnetic manipulation of microdroplets on a chip as a platform for bioanalytical applications. Sens Actuators B Chem. 2006; 117(2):457-463.

25. Li CZ, Lodder JC. The influence of the packing density on the magnetic-behavior of alumite media. J Magn Magn Mater. 1990; 88(1-2):236-246.

26. Meehan, T. Chemistry. Chapel Hill: UNC; 2007. Quantitative Magnetophoresis of Micro and Nano Particles.

27. Nielsch K, Muller F, Li AP, Gosele U. Uniform nickel deposition into ordered alumina pores by pulsed electrodeposition. Adv Mater. 2000; 12(8):582-586.

28. Okada H, Toguchi H. Biodegradable microspheres in drug-delivery. Crit Rev Ther Drug Carrier Syst. 1995; 12(1):1-99. [PubMed: 8521523]

29. Osborn JA. Demagnetizing factors of the general ellipsoid. Phys Rev. 1945; 67(11-12):351-357.

30. Powell RL, Aharonson EF, Schwarz WH, Proctor DF, Adams GK, Reasor M. Rheological behavior of normal tracheobronchial mucus of canines. J Appl Physiol. 1974; 37(3):447-451. [PubMed: 4415628]

31. Quemada D. Rheological modelling of complex fluids. Eur Phys J Appl Phys. 1998; 1:119-127.

32. Rubin BK. The pharmacologic approach to airway clearance: mucoactive agents. Paediatr Respir Rev. 2006; 7(Supplement 1):S215-S219. [PubMed: 16798570]

33. Safarik I, Safarikova M. Magnetic nanoparticles and biosciences. Monatshefte Fur Chemie. 2002; 133(6):737-759.

34. Seddon DJ, Snashall PD. Measurement of lung tissue mass in Pneumocystis carinii pneumonia. Thorax. 1989; 44(8):640-644. [PubMed: 2678578]

35. Squires TM. Nonlinear microrheology: bulk stresses versus direct interactions. Langmuir. 2008; 24(4):1147-1159. [PubMed: 18154310]

36. Tartaj P, Morales MD, Veintemillas-Verdaguer S, Gonzalez-Carreno T, Serna CJ. The preparation of magnetic nanoparticles for applications in biomedicine. J Phys D Appl Phys. 2003; 36(13):R182-R197.

37. Tirado MM, de la Torre JG. Translational friction coefficients of rigid, symmetric top macromolecules—application to circular-cylinders. J Chem Phys. 1979; 71(6):2581-2587.

38. Wust P, Hildebrandt B, Sreenivasa G, Rau B, Gellermann J, Riess H, Felix R, Schlag PM. Hyperthermia in combined treatment of cancer. Lancet Oncol. 2002; 3(8):487-497. [PubMed: 12147435]

39. Yager J, Chen TM, Dulfano MJ. Measurement of frequency of ciliary beats of human respiratory epithelium. Chest. 1978; 73(5):627-633. [PubMed: 648216]

40. Zhu X, Kundukad B, van der Maarel JR. Viscoelasticity of entangled lambda-phage DNA solutions. J Chem Phys. 2008; 129(18):185103. [PubMed: 19045431]

\section{LIST OF SYMBOLS}

a Scales width of shear thinning regime in Carreau-Yasuda model

$\vec{B} \quad$ Magnetic induction

$\boldsymbol{B}_{\mathbf{m}} \quad$ Magnitude of magnetic field

$\vec{F}_{\mathrm{d}} \quad$ Frictional drag force on particle

$\vec{F}_{\mathrm{m}} \quad$ Magnetic driving force

$\boldsymbol{L} \quad$ Cylinder length

$\boldsymbol{L}_{\mathbf{m}} \quad$ Length of drive magnet 


\begin{tabular}{ll}
$\boldsymbol{M}_{\mathbf{S}}$ & Magnetic saturation of drive magnet \\
$\vec{m}_{\mathrm{eff}}$ & Effective particle magnetization \\
$\boldsymbol{n}$ & Flow-behavior index \\
$\boldsymbol{p}$ & Aspect ratio \\
$\boldsymbol{r}$ & Radial location of fluid moving with respect to sphere \\
$\boldsymbol{r}_{\mathbf{c}}$ & Cylinder radius \\
$\boldsymbol{R}_{\mathbf{m}}$ & Radius of drive magnet \\
$\boldsymbol{r}_{\mathrm{S}}$ & Sphere radius \\
$v$ & Particle velocity \\
$\boldsymbol{V}_{\mathbf{c}}$ & Cylinder volume \\
$\boldsymbol{V}_{\mathbf{S}}$ & Sphere volume \\
$v_{\theta}$ & Azimuthal velocity \\
$z$ & Axial distance from drive magnet \\
$\beta$ & Geometry coefficient \\
$\beta_{\mathbf{c}}$ & Geometry coefficient, cylindrical geometry \\
$\beta_{\mathrm{S}}$ & Geometry coefficient, spherical geometry \\
$\eta_{\|}$ & Correction factor for cylindrical drag in axial direction \\
$\gamma_{\mathbf{s}}$ & Shear rate for sphere \\
$\gamma_{\mathbf{c}}$ & Shear rate for cylinder \\
$\eta_{\mathbf{0}}$ & Viscosity of medium at zero-shear \\
$\eta_{\mathrm{app}}$ & Apparent viscosity \\
$\theta$ & Angular location for fluid velocity \\
$\lambda$ & Thinning time constant \\
$\mu_{\mathbf{0}}$ & Permeability of free space \\
$\mu_{\mathbf{r}}$ & Relative permeability of sphere \\
& \\
\hline &
\end{tabular}




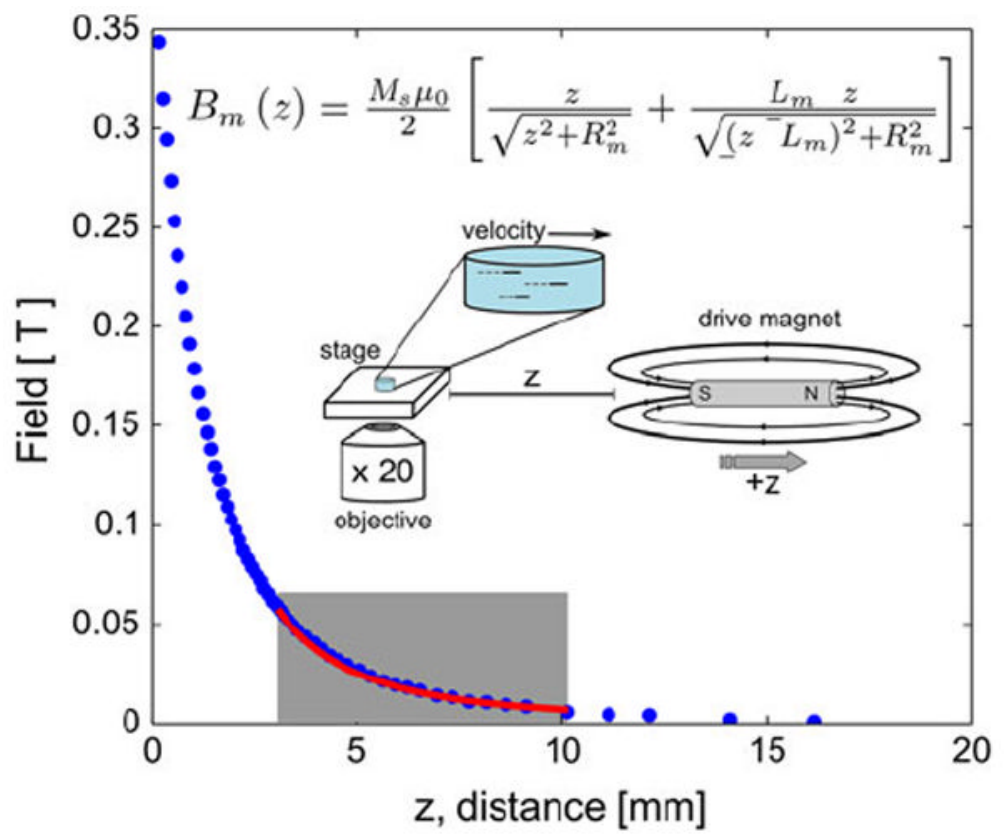

FIGURE 1.

Measured magnetic field as a function of distance from the face of the cylindrical permanent source magnet with mathematical fit (red, solid). The shaded box represents the field measurements over the experimentally relevant distance range. Inset: Apparatus. At right is the axially adjustable cylindrical magnet we use to actuate the microparticles. At left is a microscope objective where the sample volume resides within a PDMS well (top and bottom glass coverslips are not shown). The inset shows the time-lapsed paths of magnetically translating particles as a series of dots. 


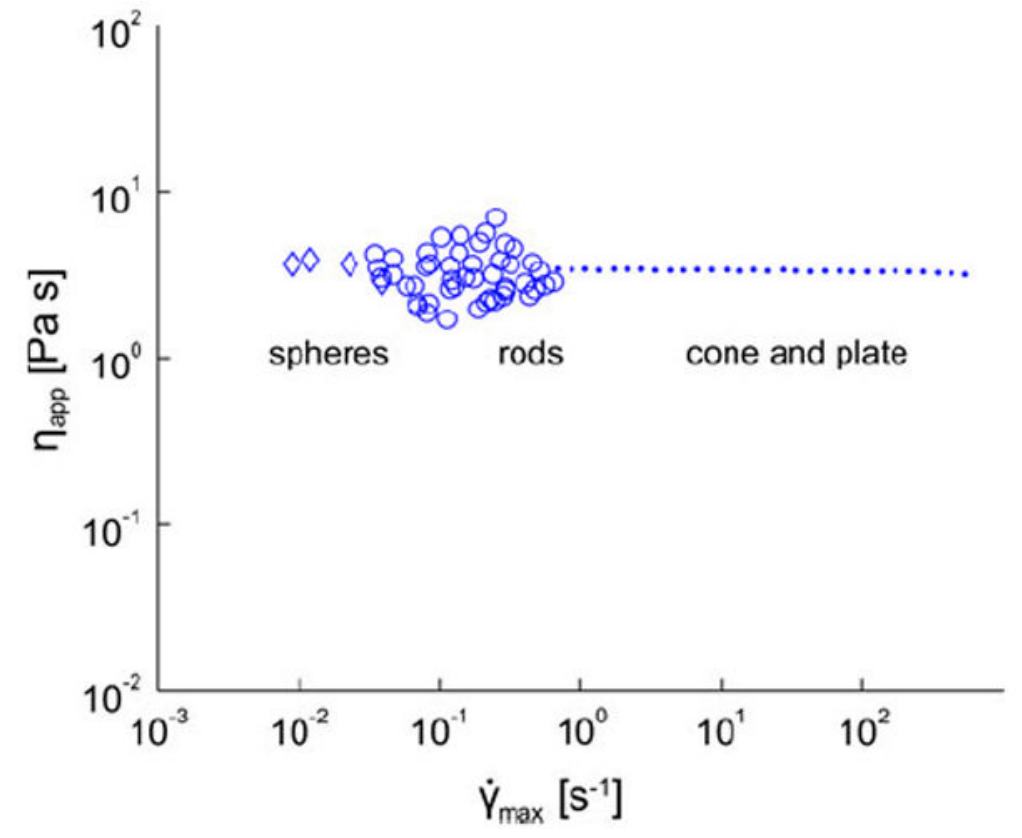

FIGURE 2.

The viscosity $\left(\eta_{\text {app }}\right)$ of the Newtonian standard solution as a function of the applied shear rate $\left(\gamma_{\max }\right)$ measured by CAP rheometry $(\cdot)$ and driven particle velocitometry (MyOne beads $\diamond$, nickel rods $\bigcirc$ ). The viscosity is constant as a function of the applied shear rate, which is indicative of Newtonian behavior. The CAP rheometer and microparticle probes both measure the same average viscosity. 


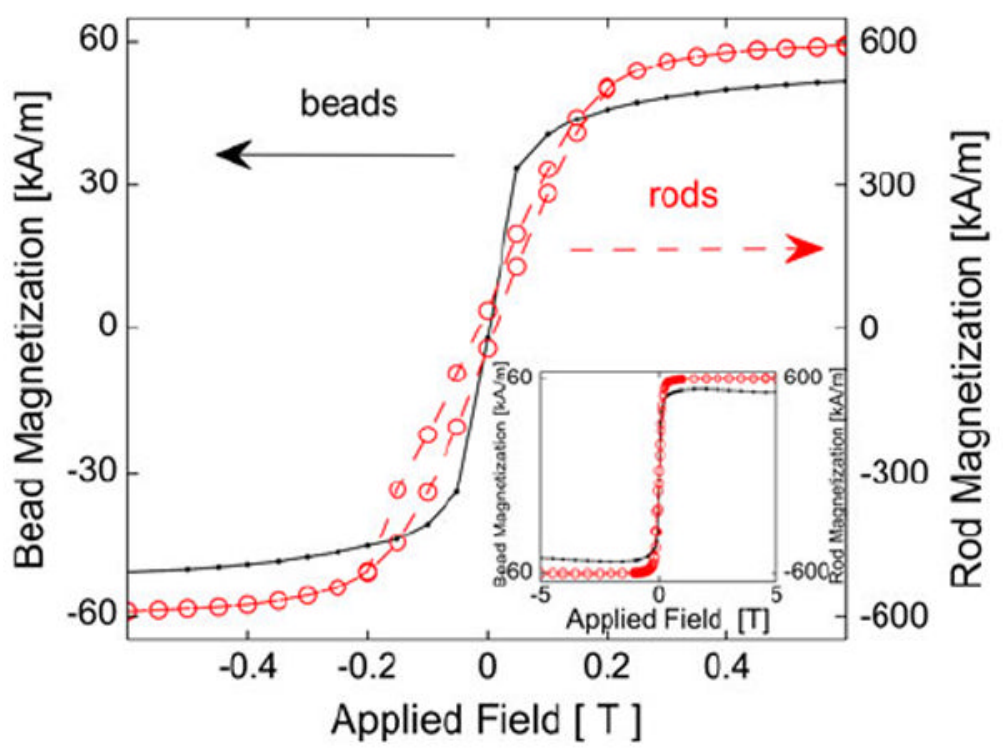

FIGURE 3.

Measured magnetization per MyOne bead or nickel nanorod long axis as a function of the applied magnetic field $( \pm 5 \mathrm{~T})$, measured at $290 \mathrm{~K}$ by SQUID magnetometry. We tested the beads as a dried suspension and the rods as a membrane-bound array. The bead polymer matrix and the sample holder induce a slight diamagnetic background that we removed by normalization. We also normalized the sample magnetization with respect to the number of particles. Beads show an absence of area inside the hysteresis loop, indicating these particles exhibit only paramagnetic behavior whereas rods show a small amount of remanent magnetization, indicating a slight ferromagnetic behavior. We define particle saturation at fields where particle magnetization reaches $95 \%$ of its maximum value. 


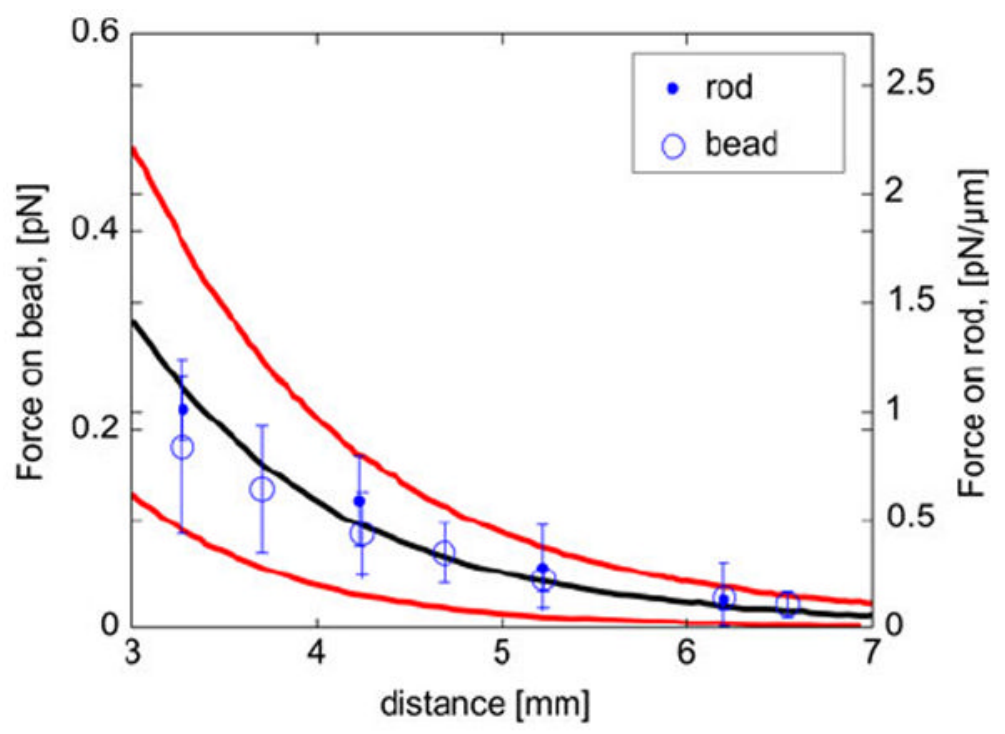

FIGURE 4.

The average magnetophoretic force applied to MyOne beads $(\bigcirc)$ and length-normalized nickel nanorods (O) plotted as functions of the distance from the permanent magnet. The bars on these data indicate the standard deviation of force as calculated based on the velocities of individual nanoparticles in the Newtonian standard solution. The black solid line plots the theoretical force imposed on the beads based on the measured field and measured magnetic properties of the nanoparticles. The red lines indicate the upper and lower bounds on the uncertainty of the theoretical force. 


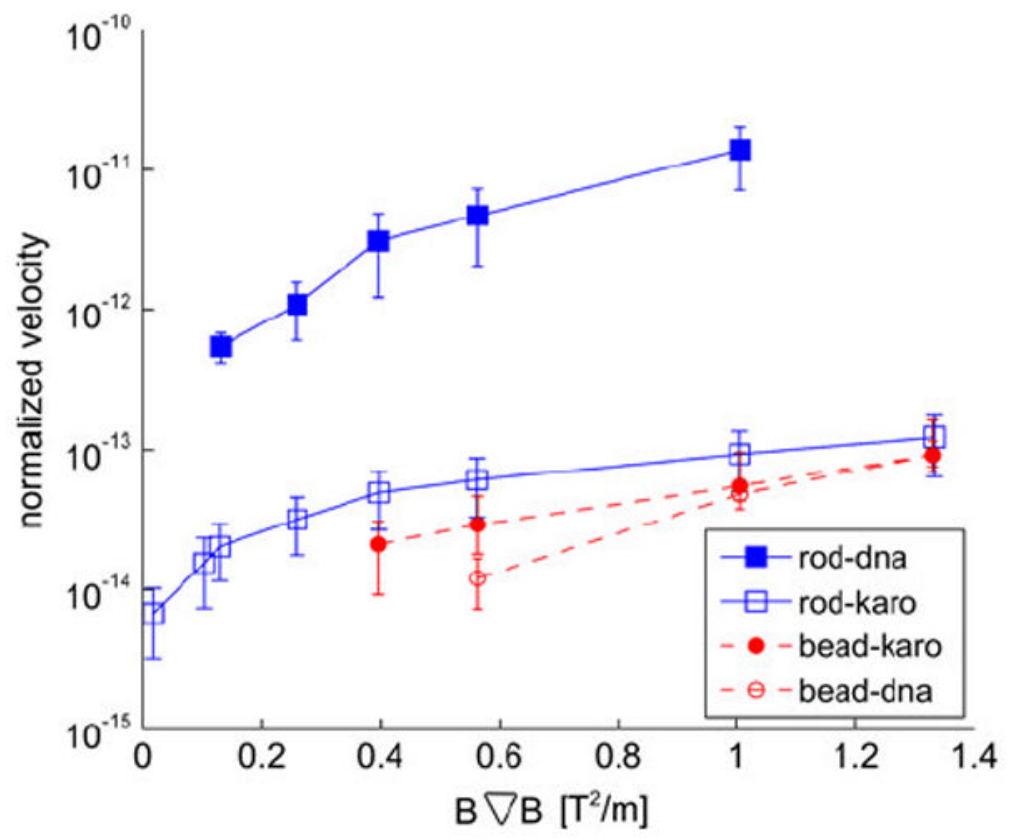

FIGURE 5.

Normalized mobilities of rods $(\square, \square)$ and MyOne beads $(\mathbf{Q}, \bigcirc)$ in the Newtonian solution (open symbols) and $0.7 \mathrm{mg} / \mathrm{mL} \lambda$-DNA solutions (closed symbols) as functions of the magnetic force proportionality $B \nabla B$. We normalized the particle mobilities with respect to their geometry-dependent drag and further normalized the rods with respect to their lengths in microns. The units of the normalized velocities for the rods and the beads are $\mathrm{m}^{2} / \mathrm{s}$ per $\mu \mathrm{m}$ length of the rod and $\mathrm{m}^{2} / \mathrm{s}$ respectively. The bars on the data points represent the range of particle velocity and do not indicate measurement error. 


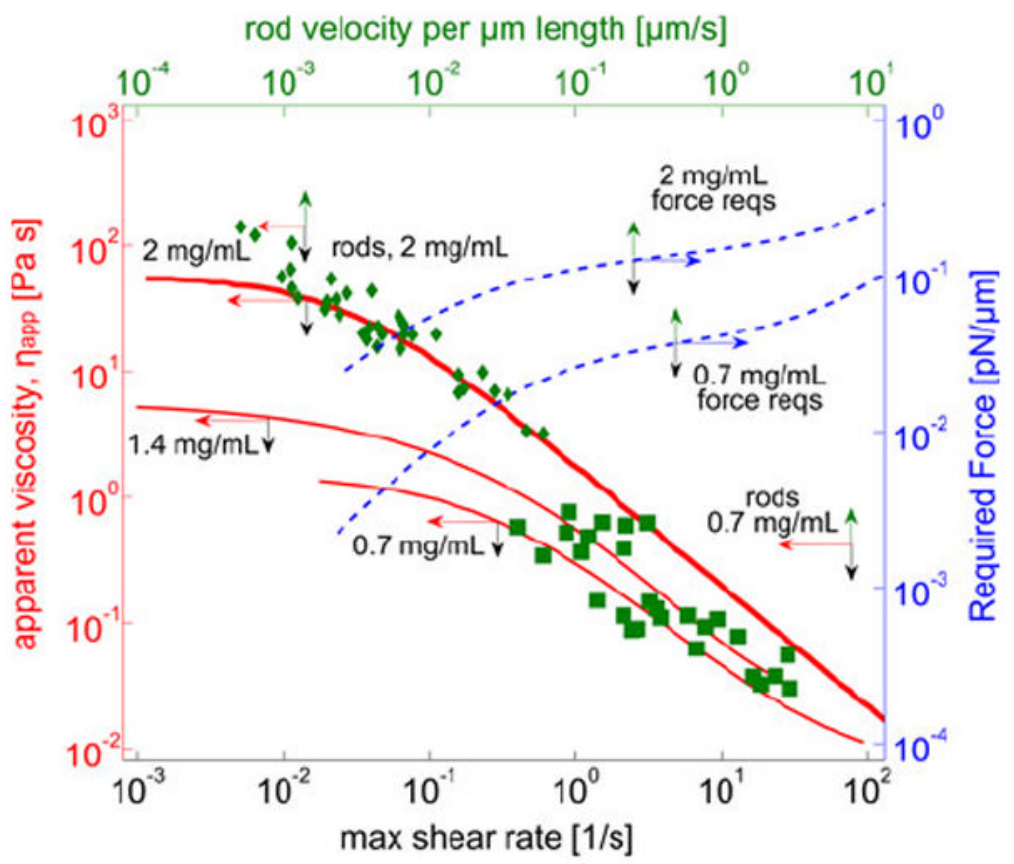

FIGURE 6.

Apparent viscosity $\left(\eta_{\text {app }}\right)$ as a function of the maximum applied shear rate $\left(\gamma_{\max }\right)$ in $\lambda$-DNA solutions. The data shown for $0.7 \mathrm{mg} / \mathrm{mL}$ as the solid, red lines are macroscale measurements from Heo and Larson. ${ }^{13}$ Corresponding lines for 1.4 and $2 \mathrm{mg} / \mathrm{mL}$ concentrations are CAP measurements by our lab and extrapolated values, respectively. Shown as discrete data points is $\eta_{\text {app }}$ as experienced by magnetophoretically driven rods ( $\square$, $\diamond$,) in $0.7 \mathrm{mg} / \mathrm{mL}$ and $2 \mathrm{mg} / \mathrm{mL}$ solutions, where the decrease in $\eta_{\text {app }}$ with increasing $\gamma_{\max }$ indicates shear thinning. Also shown are force requirements (blue lines) for translating a 1 $\mu \mathrm{m}$ long rod with a $100 \mathrm{~nm}$ diameter through 0.72 and $1.44 \mathrm{mg} / \mathrm{mL}$ DNA solutions as a function of shear rate. Because the DNA solution shear thins, small increments in force yield much higher particle velocities than expected because of the shear thinning effect. 
TABLE 1

Carreau parameters for $\lambda$-DNA test solutions.

\begin{tabular}{llll}
\hline DNA Conc. $(\mathbf{m g} / \mathbf{m L})$ & $\mathbf{0 . 7}$ & $\mathbf{1 . 4}$ & $\mathbf{2 . 0 ^ { \boldsymbol { a } }}$ \\
$\eta_{0}(\mathrm{cP})$ & 1300 & 3900 & 17000 \\
$\eta_{\infty}(\mathrm{cP})$ & 5 & 8 & 11 \\
$\lambda(\mathrm{s})$ & 5.7 & 7.1 & 16 \\
$n$ & 0.15 & 0.038 & 0.034 \\
$a$ & 1.3 & 1.0 & 0.8 \\
Testing regime & Driven microparticles & CAP & Driven microparticles \\
\hline
\end{tabular}

${ }^{a}$ Extrapolated from Heo and Larson ${ }^{13}$ and $1.4 \mathrm{mg} / \mathrm{mL}$ CAP data. 


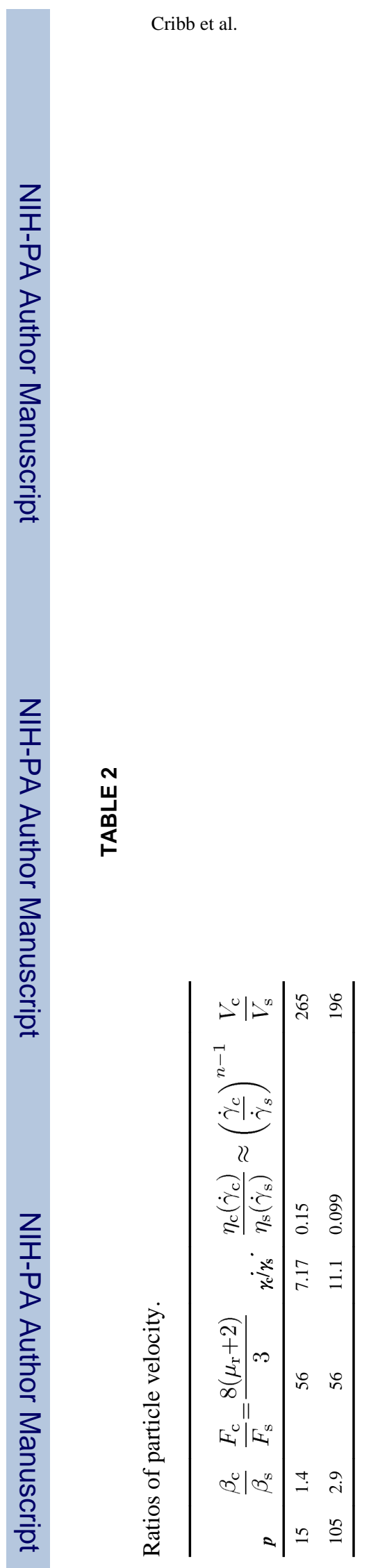

Ann Biomed Eng. Author manuscript; available in PMC 2013 December 10. 